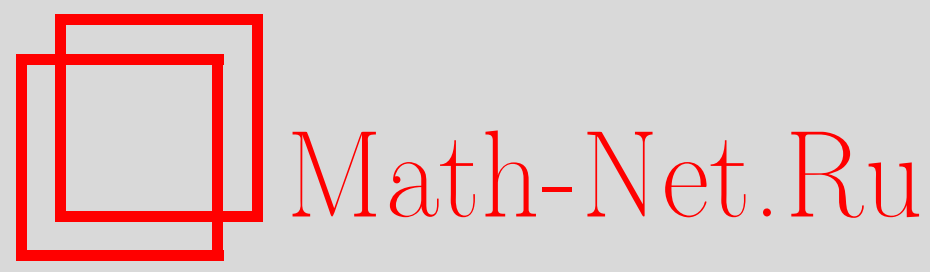

В. В. Семин, Исследование поведения триангуляции на симплициальных комплексах, Дискрет. матем., 2011, том 23, выпуск 1, 119-131

DOI: https://doi.org/10.4213/dm1135

Использование Общероссийского математического портала Math-Net.Ru подразумевает, что вы прочитали и согласны с пользовательским соглашением http://www . mathnet.ru/rus/agreement

Параметры загрузки:

IP : 54.80 .73 .141

26 апреля 2023 г., 14:46:08 


\title{
Исследование поведения триангуляции на симплициальных комплексах
}

\author{
(c) 2011 г. $\quad$ В. В. Семин
}

\begin{abstract}
Задача представления топологических моделей в виде триангулированных кубических комплексов связана с характером и поведением триангуляции в $n$-мерном пространстве. Основой для анализа данных особенностей может служить фундаментальная статистическая информация, позволяющая производить синтез характерных особенностей триангуляции в модельных пространствах различной размерности. В данной статье предложен подход к решению этой задачи на примере анализа статистических особенностей триангуляции для пространств размерности не выше чем 4 при помощи бинарного кодирования.
\end{abstract}

\section{1. Введение}

Рассмотрим множество всех целых точек $n$-мерного евклидова пространства $\mathbf{Z}^{n}$, то есть точек с целочисленными координатами. Будем рассматривать случаи с $n \leqslant 4$. Определим решеточную структуру соответствующей размерности как множества всех вершин из $\mathbf{Z}^{2}$, $\mathbf{Z}^{3}, \mathbf{Z}^{4}$ и множества простых ребер-отрезков $V_{p}$ нормы $p$, инцидентных вершинам, где $p=\max \left|X_{i}\right|, i=1, \ldots, n$, если $\chi=\left(X_{1}, \ldots, X_{n}\right)-$ вектор ребра. Тогда решеточная структура образует неориентированный граф, каждая вершина которого является целой точкой. Собственно пару $\left(\mathbf{Z}^{n}, V_{p}\right)$, где $p, n-$ натуральные числа, будем называть решеткой. Если потребовать, чтобы евклидова длина простого ребра из $V_{p}$ не превышала единицы, то есть $p=1$, то для пространств размерности не выше 4 это будет означать построение решетки соответствующей размерности. Так для пространства размерности 2 граф будет представлять собой простую решетку, для пространства размерности 3 трехмерную решетку с единичным кубом в основе и так далее. Определение решеточной структуры, данное выше, следует логике определений, приводимых в [1].

Для каждой такой решетки, покрывающей пространство соответствующей размерности можно задать ее триангуляцию, как проведение диагонали в каждой двумерной грани. Полученная таким образом триангуляция тривиальна для пространства размерности 2, но для пространств высшей размерности триангуляция может называться правильной, только если она приводит к разбиению всей структуры на множество правильно расположенных симплексов. Основой такой решетки может служить $n$-мерный куб $I^{n}$. Процесс правильной триангуляции $I^{n}$ приводит к выделению ограниченного набора конфигураций, которые служат основой для триангуляции $I^{n+1}$. При такой модели триангуляции достигается эргодичность поведения триангуляции во всем пространстве, что дает возможность на основе поведения триангуляции $I^{n}$ сделать вывод о поведении триангуляции всего пространства. 
В настоящей статье проводится

- построение правильной триангуляции куба, как элемента решетки множеств $\mathbf{Z}^{2}, \mathbf{Z}^{3}$ и $\mathbf{Z}^{4}$;

- выделение типов правильно триангулированных кубов и частоты (вероятности) их появления при стохастической триангуляции пространства;

- выделение статистических особенностей и распределений полученных частот;

- анализ полученных результатов и построение общего статистического метода анализа на основе распределения типов правильно триангулированных кубов в описанных выше пространствах размерности не выше, чем 4.

\section{2. Триангуляция в двумерном пространстве}

Для построения триангуляции двумерной решетки $\left(\mathbf{Z}^{2}, V_{1}\right)$ с узлами в целых точках может послужить модель плотного плоского покрытия. Приведем его определение.

Определение 1. Будем называть плотным плоским покрытием $P_{k}^{2}$ множество

$$
P=\{1 \leqslant x \leqslant X, 1 \leqslant y \leqslant Y\}
$$

с простыми ребрами индекса $k$ и таким соединением ребрами-отрезками всех целых точек из $P$, когда нет ни одного пересечения ребер-отрезков и нельзя добавить ни одного дополнительного ребра без получения пресечения. Здесь индекс $k$ означает, что для любого ребра-отрезка с координатами $\left\{\left(x_{0}, y_{0}\right),\left(x_{1}, y_{1}\right)\right\}$ выполняются соотношения

$$
\left|x_{0}-x_{1}\right| \leqslant k, \quad\left|y_{0}-y_{1}\right| \leqslant k,
$$

и $\left|x_{0}-x_{1}\right|,\left|y_{0}-y_{1}\right|$ взаимно просты.

Точнее, мы будем рассматривать покрытие $P_{1}^{2}$, приведенное на рис. 1 .

Очевидно, что такая триангуляция тривиальна. В данной решетке можно выделить всего два вида ячеек - клетку с диагональю, ориентированной влево, и клетку с диагональю, ориентированной вправо. Такая структура легко кодируется бинарным кодом, состоящим из 0 и 1. Единица записывается в соответствующую ячейку, если диагональ имеет правую ориентацию и ноль, если диагональ имеет левую ориентацию.

При задании стохастической триангуляции на решетке $\left(\mathbf{Z}^{2}, V_{1}\right)$ покрытие $P_{1}^{2}$ может служить моделью триангуляции последнего благодаря эргодичности поведения триангуляции во всем пространстве $\mathbf{Z}^{2}$. Эргодичность следует из того, что возможны всего два состояния клетки 0 и 1 , а $P_{1}^{2}$ является частью триангулированной решетки $\left(\mathbf{Z}^{2}, V_{1}\right)$ как пространства. Очевидно, что при такой триангуляции, вероятность получить в произвольно выбранной клетке $P_{1}^{2}$ значения 0 или 1 равна $1 / 2$. Таким образом, оба состояния клетки равновероятны при задании стохастической триангуляции.

\section{3. Триангуляция в трехмерном пространстве}

Рассмотрим множество $\left(\mathbf{Z}^{3}, V_{1}\right)$, где вершины являются узлами трехмерной целочисленной решетки, а множество простых ребер-отрезков имеет норму $p=1$, образуя множество ребер решетки инцидентных вершинам. Основным элементом полученной структуры является единичный куб $I^{3}$ с вершинами в целых точках и простыми ребрами-отрезками в качестве одномерных граней. 


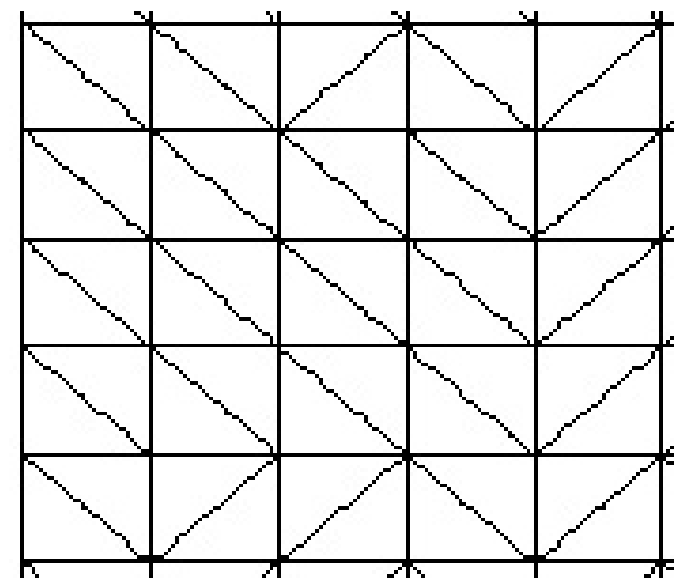

Рис. 1. Покрытие $P_{1}^{2}$

Определение 2. Определим произвольную (стохастическую) триангуляцию на множестве $\left(\mathbf{Z}^{3}, V_{1}\right)$ как триангуляцию двумерных граней трехмерной решетки, получаемую проведением диагонали в соответствующей грани.

Введем понятие правильной триангуляции, основывающееся на требовании правильного расположения симплексов по Понтрягину [2].

Определение 3. Триангуляцию будем называть правильной, если она порождает разбиение выбранного пространства на множество правильно расположенных симплексов по Понтрягину.

Очевидно, что произвольная триангуляция $\left(\mathbf{Z}^{3}, V_{1}\right)$ не всегда будет правильной, то есть будут существовать такие триангуляции двумерных граней $I^{3}$, которые не порождают разбиения куба на правильно расположенные симплексы.

Опишем формально требование правильного разбиения $I^{3}$ на правильно расположенные симплексы. Рассмотрим вектор диагональных индексов вершин $x=\left(x_{0}, x_{1}, x_{2}, x_{3}\right)$, где $x_{i}$ - целое неотрицательное число - число вершин, имеющих $0,1,2$ и 3 инцидентных диагоналей соответственно.

Тогда необходимым условием выполнения требования триангуляции $I^{3}$ будет выполнение системы двух диофантовых уравнений

$$
\sum_{i=0}^{3} x_{i}=8, \quad \sum_{i=0}^{3} i x_{i}=12 .
$$

Вектор диагональных индексов вершин будет являться решением системы, однозначно характеризуя тип триангуляции $I^{3}$.

Очевидно, рассматриваемой системе, с учетом требования разбиения куба на правильно расположенные симплексы, удовлетворяют следующие наборы решений:

(1) $x=(0,6,0,2)-1$ тип,

(2) $x=(1,3,3,1)-2$ тип, 


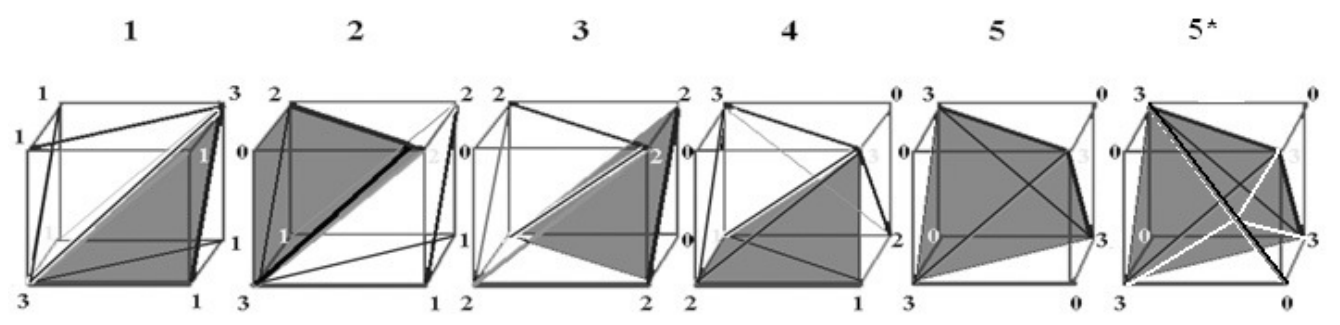

Рис. 2. Типы правильной триангуляции $I^{3}$

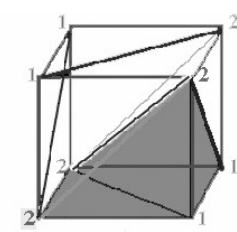

6

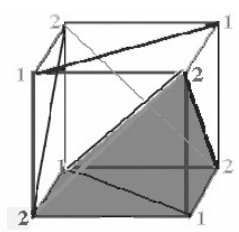

7

Рис. 3. Типы неправильной триангуляции $I^{3}$

(3) $x=(2,0,6,0)-3$ тип,

(4) $x=(2,2,2,2)-4$ тип,

(5) $x=(4,0,0,4)-5$ тип.

Таким образом, соответствие конфигурации куба одному из вышеперечисленных 5 типов является достаточным условием выполнения требования правильной триангуляции. На рис. 2 приведено графическое изображение всех пяти типов.

Типы неконгруэнтны, и других правильных триангуляций $I^{3}$ не существует. Полная триангуляция $I^{3}$ для типов 1-4 завершается проведением большой диагонали внутри куба. При этом положение ее однозначно для 1-го, 2-го и 4-го типов. Третий тип допускает три различных положения большой диагонали, в то время как триангуляция граней остается неизменной. При триангуляции 5-го типа большая диагональ внутри куба может проводиться (рис. $2\left(5^{*}\right)$ ), а может быть не проведена (рис. 2 (5)), причем ее ориентация или отсутствие не влияет на триангуляцию.

Однако системе (а) удовлетворяет также решение $(0,4,4,0)$. Данный вектор порождает неоднозначную триангуляцию двумерных граней $I^{3}$, что приводит к двум новым типам 6 и 7 (рис. 3), которые не удовлетворяют требованию разбиения куба на правильно расположенные симплексы. Эти типы будем считать неполнотриангулированными.

Цифрами в вершинах кубов на рис. 3 обозначено число диагональных ребер, инцидентных данной вершине. Исходя из вышеприведенных определений и рассуждений, легко видеть, что стохастическая триангуляция $\left(\mathbf{Z}^{3}, V_{1}\right)$ порождает разбиение кубов решетки в соответствии с одним из 7 типов.

Полную информацию о поведении триангуляции в данном множестве можно получить, построив распределение по типам, встречающимся при произвольной триангуляции $I^{3}$. 


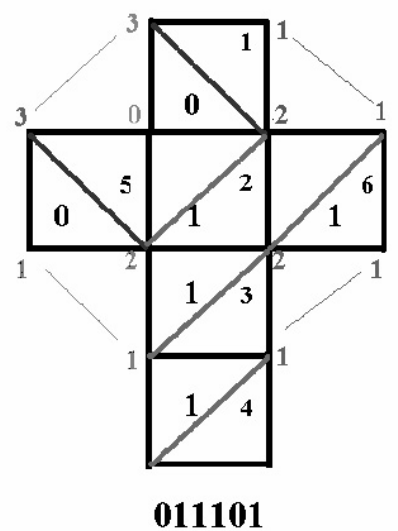

Рис. 4. Развертка куба с двоичным кодом (011101)

Для этого закодируем каждую возможную конфигурацию шестью двоичными разрядами в соответствии с числом двумерных граней куба. Не уменьшая общности рассуждений, зафиксируем порядок развертки куба. Будем считать, что 0 записывается в соответствующий разряд, если диагональ грани имеет левую ориентацию и 1 в противном случае. Пример подобной развертки показан на рис. 4.

Тогда каждой такой развертке может быть однозначно сопоставлен вектор

$$
\alpha=\left(a_{1}, a_{2}, a_{3}, a_{4}, a_{5}, a_{6}\right), \quad a_{i}=\{1,0\}, \quad i=1, \ldots, 6 .
$$

Применяя такой способ кодирования, легко определить число всех возможных конфигураций $I^{3}-2^{6}$. При этом каждый такой код однозначно соответствует одному из 6 решений системы (а). Очевидно, что разные бинарные коды, могут соответствовать одному типу триангуляции. Поэтому необходима общая система кодирования на основе числа диагональных ребер, инцидентных вершинам куба.

Примером такой системы кодирования может служить набор решений системы (a), далее система кодирования будет расширена для случая пространства размерности 4.

Рассмотрим множество элементарных событий $\Omega$ с элементами - кодами развертки. Мощность $\Omega$ составляет $2^{6}$. Определим сигма-алгебру $A$ случайных событий, как множество событий появления куба определенного типа, тогда $A=\left\{A_{1}, \ldots, A_{7}\right\}$. Вероятностную меру $\mathbf{P}$ определим стандартным образом, полагая $\mathbf{P}(\Omega)=1$. Так как множество элементарных событий имеет небольшую мощность, то искомые вероятности можно получить простым перечислением всех событий. В результате несложных вычислений получаем следующую картину. Среди всех возможных исходов (64 элементарных события) только 48 являются случаями появления правильно триангулированного куба, а 18 неполно триангулированного. Таким образом, общая вероятность получить правильную триангуляцию $I^{3}$ составляет $p=0,71875$, в то время как на долю неполной триангуляции приходится $q=0,28125$.

При стохастической триангуляции это означает, что именно с такими вероятностями можно получить ту или иную триангуляцию куба в выбранной ячейке трехмерной ре- 


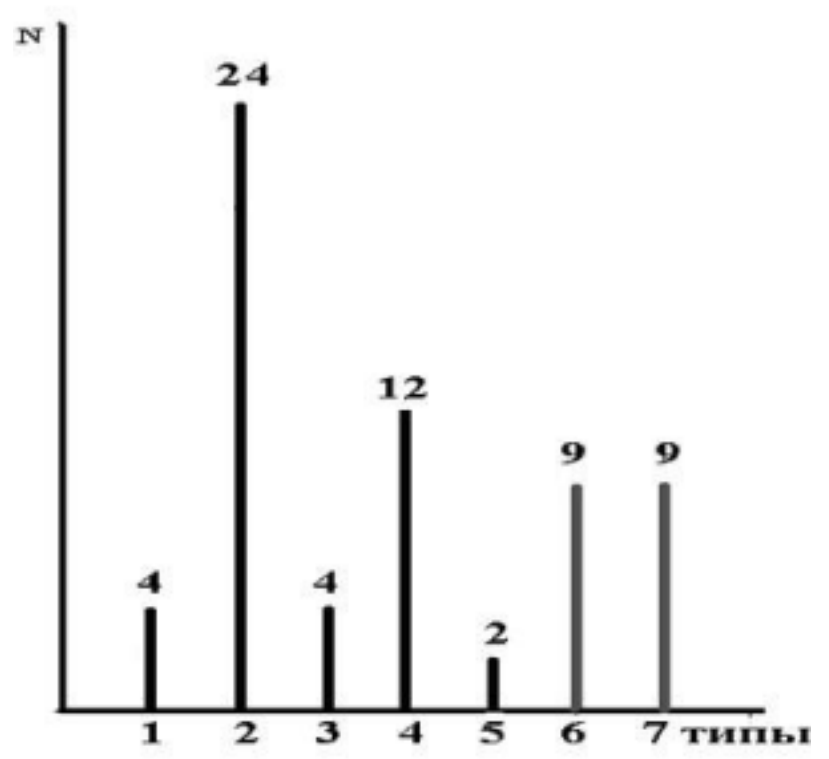

Рис. 5. Распределение по типам

шетки. Однако полное представление о поведении триангуляции на множестве $\left(\mathbf{Z}^{3}, V_{1}\right)$ может дать только распределение по типам кубов с указанием веса (вероятности события $A_{i}$ ). Графически распределение представлено на рис. 5 , где по оси абсцисс указаны типы соответствующих триангуляций кубов, а по оси ординат отложено число событий $A_{i}$, где $i-$ номер триангуляции в соответствии с классификацией, предложенной в этой статье. При рассмотрении распределения, видно, что наиболее часто встречается второй тип триангуляции, вдвое меньший вес у триангуляции четвертого типа. При этом веса остальных типов значительно ниже. Это дает основания утверждать, что наиболее часто при стохастической триангуляции будут встречаться эти типы. Неполно триангулированные типы имеют одинаковый вес, что естественно, так как они соответствуют, одному вектору диагональных индексов вершин. Суммарная вероятность появления неполных типов имеет тот же порядок, что и доминирующие два типа правильной триангуляции $I^{3}$. Следовательно, данные типы будут встречаться также часто.

Анализ всех бинарных кодов $\alpha$ показывает, что для каждого из 5 типов $I^{3}$ можно описать вдвое меньшим числом кодов, чем число событий $A_{i}$, представленных на рис. 5 , так как для любого кода $\alpha$ соответствующего некому типу $I^{3}$ всегда найдется код $\bar{\alpha}$, соответствующий тому же типу $I^{3}$.

Сформулируем это свойство в виде утверждения.

Предложение 1. Для каждого набора

$$
\alpha=\left(a_{1}, a_{2}, a_{3}, a_{4}, a_{5}, a_{6}\right), \quad a_{i}=\{1,0\},
$$

соответствующего одному из типов триангуляиии $I^{3}$, сущеествует набор $\bar{\alpha}$, однозначно соответствующий тому же типу триангуляиии $I^{3}$. 


\section{4. Триангуляция в четырехмерном пространстве}

Теперь перейдем к рассмотрению четырехмерного пространства. Из-за повышения размерности усложняется не только наглядное представление топологических конструкций, но и поведение триангуляции в них, а также связанные с этим вычисления.

Рассмотрим множество $\left(\mathbf{Z}^{4}, V_{1}\right)$, где вершины являются узлами четырехмерной целочисленной решетки, а множество простых ребер-отрезков имеет норму $p=1$, образуя множество ребер решетки, инцидентных вершинам. Основным элементом полученной структуры является единичный куб $I^{4}$ с вершинами в целых точках и простыми ребрамиотрезками в качестве одномерных граней.

Приведенное выше определение аналогично тому, что давалось при рассмотрении трехмерного случая, за исключением поправок на размерность. По аналогии зададим произвольную (стохастическую) триангуляцию на множестве $\left(\mathbf{Z}^{4}, V_{1}\right)$, как триангуляцию двумерных граней четырехмерной решетки, получаемую проведением диагонали в соответствующей грани.

Понятие правильной триангуляции, основывающееся на требовании правильного расположения симплексов по Понтрягину, оставим в приведенной ранее формулировке без изменений.

Таким образом, неполной будет называться такая триангуляция, в которой в развертку входит хотя бы один неполно триангулированный трехмерный куб.

Как и в трехмерном случае, очевидно, что произвольная триангуляция $\left(\mathbf{Z}^{4}, V_{1}\right)$ не всегда будет являться правильной. В тоже время классификация правильных типов триангуляции $I^{4}$ достаточно велика, чтобы можно было наглядно, как для $I^{3}$, привести ее в графическом виде. В данной статье предложен способ классификации на основе вектора количества типов $I^{3}$, входящих в развертку $I^{4}$. Формально $x=\left(x_{0}, x_{1}, x_{2}, x_{3}, x_{4}\right)$, где $x_{i}$ - число трехмерных кубов $(i+1)$-го типа, входящих в развертку четырехмерного куба. При таком методе классификации существует 214 различных неконгруэнтных правильных типов триангуляции $i^{4}$.

Получим для них статистическую информацию, аналогичную распределению для типов $I^{3}$.

Четырехмерный куб имеет 24 двумерные грани, являющиеся одновременно гранями трехмерных кубов его развертки. Фиксируем порядок развертки, тогда каждую конфигурацию можно закодировать 24 двоичными разрядами, 0 записывается в соответствующий разряд, если диагональ двумерной грани ориентирована влево и 1 если вправо. По аналогии с $I^{3}$ будем сопоставлять каждой такой развертке вектор $\alpha=\left(a_{1}, a_{2}, \ldots, a_{24}\right)$, где $a_{i}=\{1,0\}, i=1, \ldots, 24$. На рис. 6 представлена трехмерная модель развертки $I^{4}$, развертка каждого куба производится аналогично трехмерному случаю (см. рис. 4).

Тогда число всех возможных конфигураций $I^{4}$ равно $2^{24}$, то есть равно числу всех двоичных векторов $\alpha$ длины 24 .

Очень важно, что любую сколь угодно сложную типологически конфигурацию можно представить с помощью бинарного кода, что упрощает рассуждения в дальнейшем.

Рассмотрим множество элементарных событий $\Omega$ с элементами - кодами развертки.

Мощность $\Omega$ равна $2^{24}$. Определим сигма-алгебру $A$ случайных событий как множество событий появления $I^{4}$ определенного типа, тогда $A=\left\{A_{1}, \ldots, A_{215}\right\}$, где $A_{215}-$ обобщенное событие появления неполной триангуляции $I^{4}$, а $\mathbf{P}(\Omega)=1$.

Введя таким образом вероятностное пространство, получим искомые вероятности событий и распределение по типам. Все расчеты, приведенные ниже, выполнены на ЭВМ с применением оптимизированных переборных алгоритмов. 


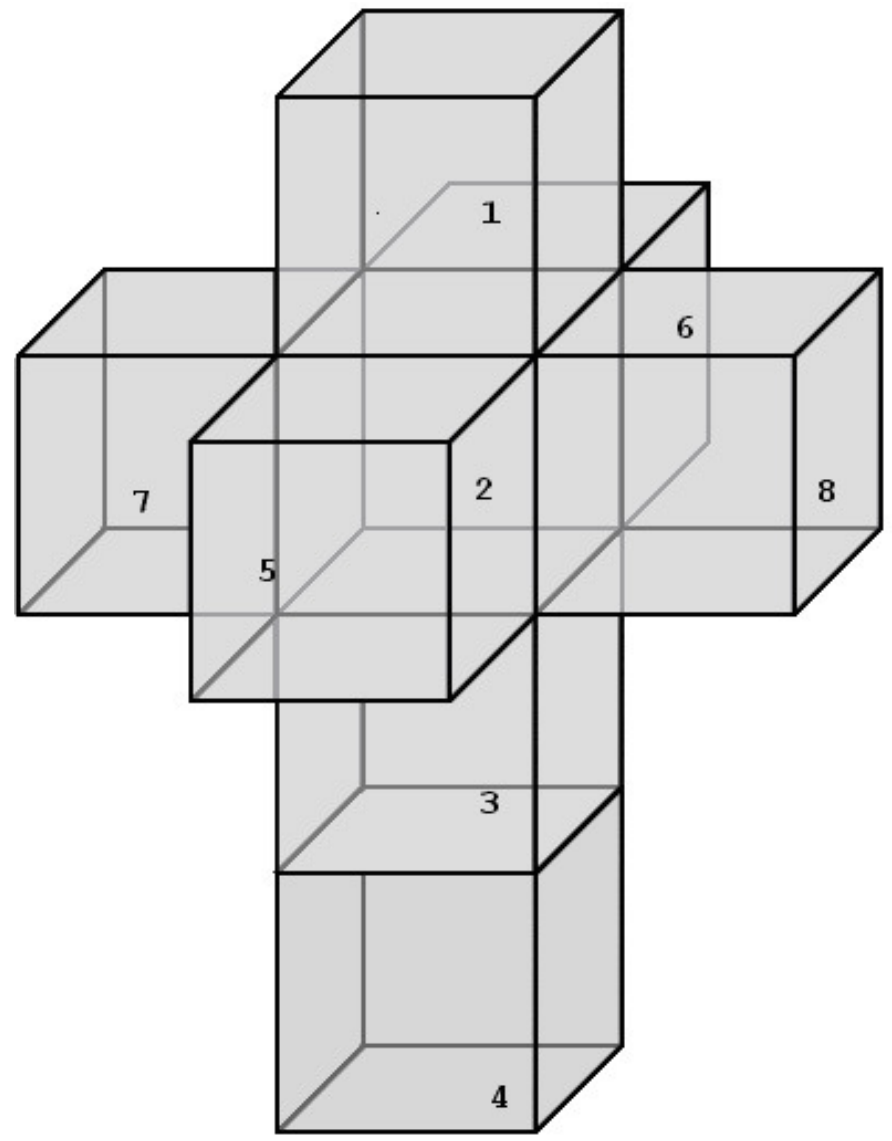

Рис. 6. Развертка четырехмерного куба

Число перебранных вариантов равно 16777 216, что соответствует числу всех возможных бинарных кодов длины 24, где 24 - число двумерных граней четырехмерного куба. Число всех правильных конфигураций $I^{4}$ равно 1099 404, соответственно число всех неполных конфигураций равно 15677812.

Таким образом, вероятность выделить правильно триангулированный четырехмерный куб в трехмерном пространстве составляет 0,06553913, неполно триангулированный - 0,93446087. По сравнению с трехмерным пространством, вероятность получить правильную триангуляцию $I^{4}$ ничтожно мала, что дает основания утверждать, что при повышении размерности эта вероятность будет только уменьшаться. При этом число различных правильно триангулированных конфигураций $I^{4}$ равно 56598 . На рис. 7 наглядно отображено общее распределение $I^{4}$ по типам.

Распределение называется общим в силу принципа классификации типов $I^{4}$, так как соотнесение с типом происходит исходя из максимального числа типов трехмерных кубов, присутствующих в развертке четырехмерного куба. Например, Тип 1 - все конфигурации четырехмерного куба, в которых в развертке преобладает 1-й тип правильно триангулированного трехмерного куба. 


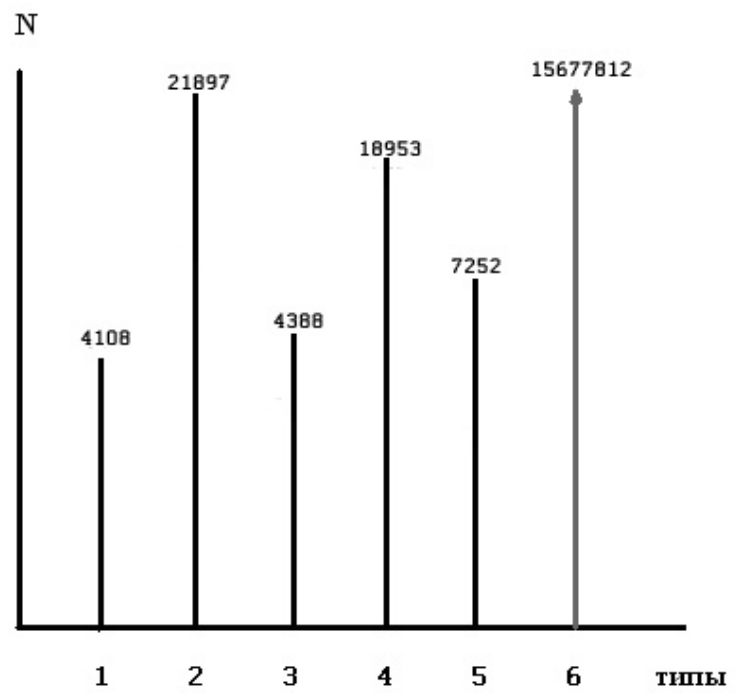

Рис. 7. Общее распределение по типам $I^{4}$

Очевидно, что такая классификация неоднозначна, в силу того, что в развертке могут находиться в одинаковом количестве несколько различных типов кубов.

Пример такой развертки - (245421 13), где значение каждой позиции соответствует типу трехмерного куба развертки.

Приведенное на рис. 7 распределение отличается по методу классификации от предложенного выше в данной статье. При его рассмотрении видно, что в развертках преобладают два типа (второй и четвертый), что подтверждает результаты, полученные при построении распределения типов триангуляций для $I^{4}$.

На рис. 8 приведено изображение триангулированной развертки $I^{4}$, целиком состоящей из кубов первого типа и вектором числа типов $(8,0,0,0,0)$.

К сожалению, распределение, изображенное на рис. 7, не может дать точную статистическую картину поведения триангуляции из-за неоднозначности и сильной обобщенности. Более детальную картину можно получить, построив распределение по векторам числа типов. Как было отмечено ранее, при использовании такой классификации существует 214 различных неконгруэнтных правильных типов триангуляции $I^{4}$.

Оценим общее число $N$ всевозможных векторов числа типов. Напомним, что вектор выглядит следующим образом: $x=\left(x_{0}, x_{1}, x_{2}, x_{3}, x_{4}\right)$, где $x_{i}$ - число кубов $(i+1)$-го типа, входящих в развертку четырехмерного куба. При этом выполняется равенство

$$
\sum_{i=0}^{4} x_{i}=8 .
$$

Пусть имеется $m$ ячеек. Нужно найти, сколькими способами можно распределить $n$ одинаковых частиц в данных ячейках. При этом $m$ ячеек разделены $m-1$ перегородкой. Тогда рассматриваемая система состоит из $n+m-1$ элементов. Общее число возможных 


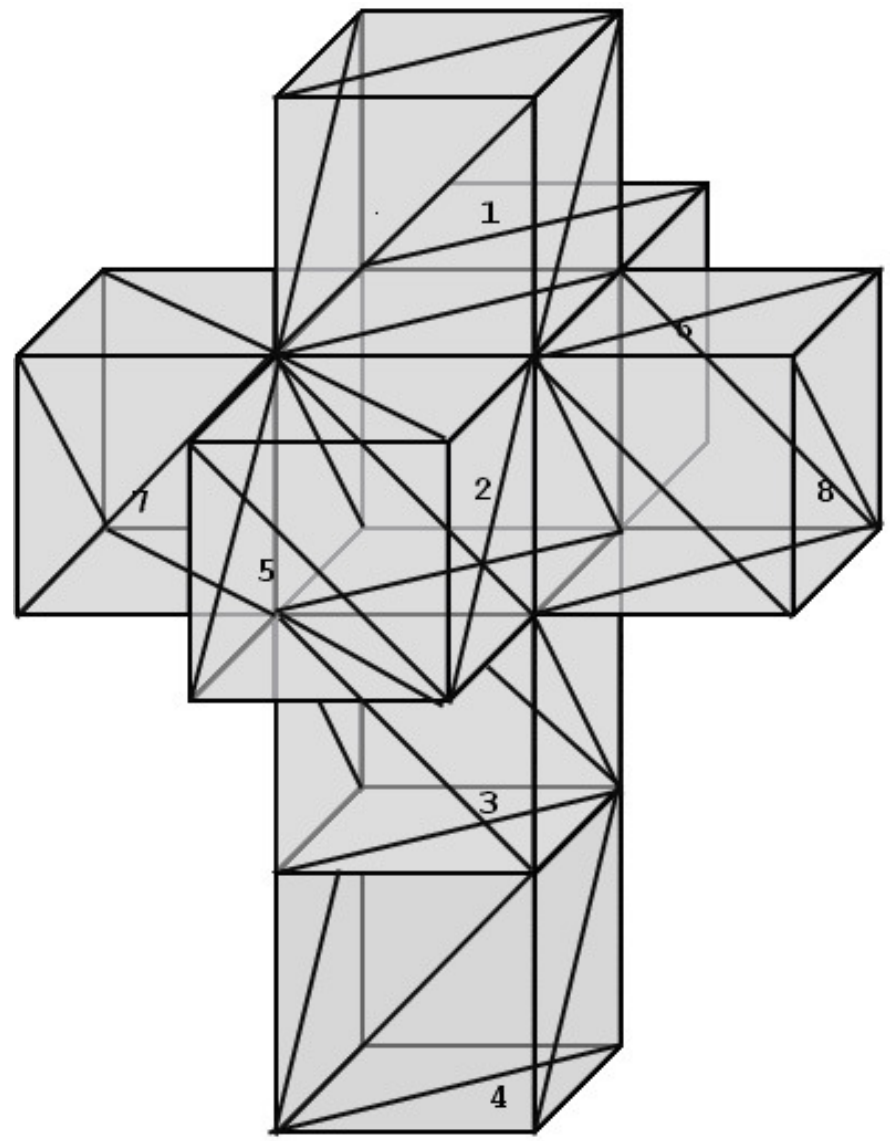

Рис. 8. Триангулированная развертка $(8,0,0,0,0)$

перестановок для такой системы равно $(n+m-1)$ !. Очевидно также, что не все перестановки приводят к новым распределениям. Перестановки частиц не изменяют состояния системы, так как частицы неразличимы. Их число равно $n$ !. Перестановки перегородок ячейки также не порождают новых состояний, их число равно $(m-1)$ !.

Таким образом, число $N$ способов размещения $n$ частиц по $m$ ячейкам равно

$$
\frac{(n+m-1) !}{n !(m-1) !}
$$

Тогда из выведенной формулы следует, что в рассматриваемом случае это число равно

$$
\frac{(8+4) !}{8 ! 4 !}
$$

По определению, распределение $n$ одинаковых частиц по $m$ ячейкам, называется статистической моделью Бозе-Эйнштейна [3]. В данном случае оно полностью подходит для описания распределения 8 частиц по 5 ячейкам-элементам вектора числа типов. Следовательно, построенное распределение по векторам числа типов описывается статистической моделью Бозе-Эйнштейна. 


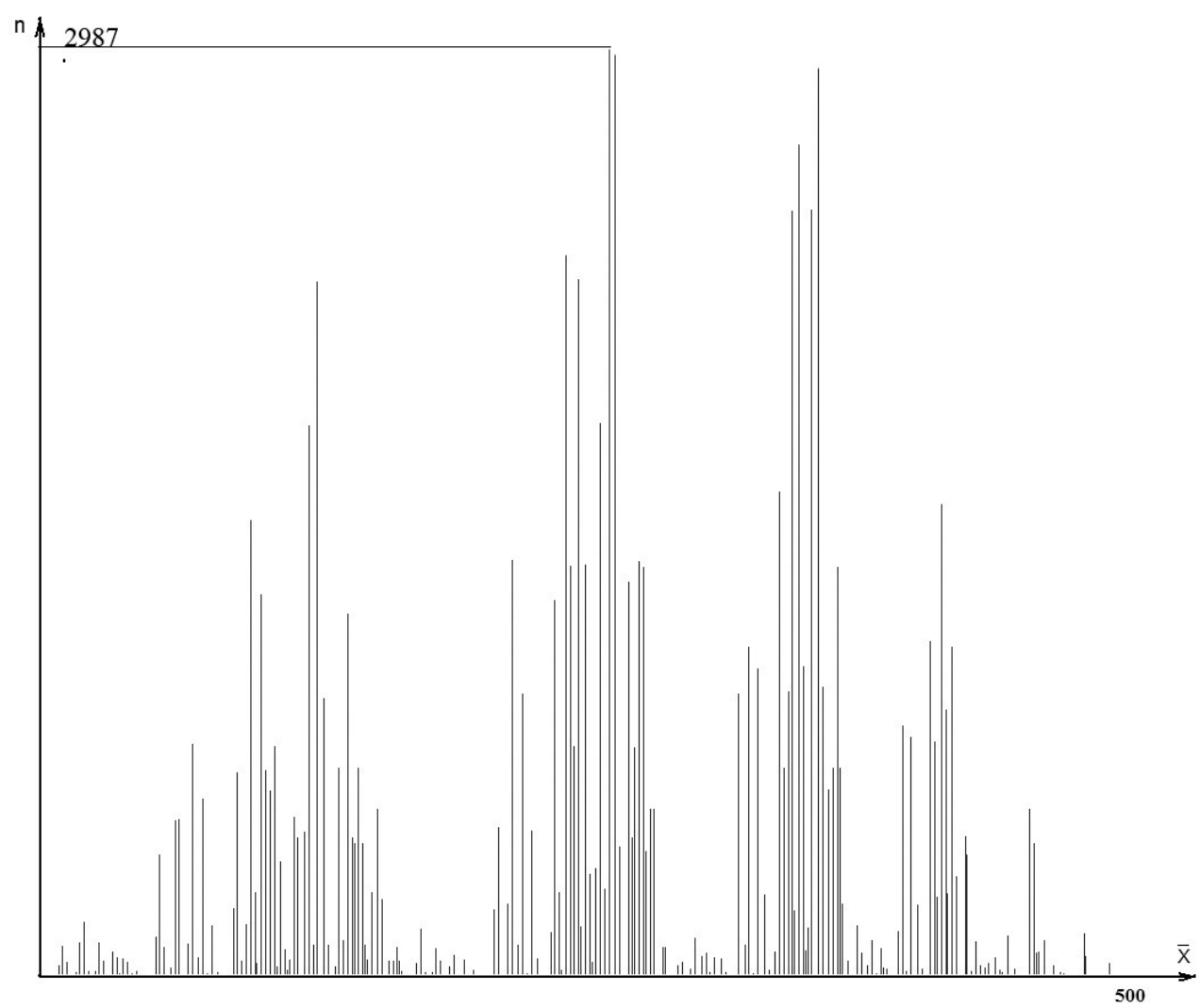

Рис. 9. Распределение по векторам числа типов

Если упорядочить все $N$ векторов в лексикографическом порядке, то распределение будет иметь вид, представленный на рис. 9. Промежутки между вертикальными линиями свидетельствуют об отсутствии правильной триангуляции $I^{4}$, соответствующей данному вектору. При общем рассмотрении становится заметно, что наибольшие веса в распределении имеют векторы с большим значением $x_{i}$, и основная их масса сосредоточена между векторами $(2,1,3,2,0)$ и $(4,4,0,0,0)$. Максимум приходится на вектор $(1,3,1,2,1)$ с весом 2987. Необходимо отметить, что похожие результаты были представлены в работе [4], однако их проверка автором показала, что в них допущены ошибки, которые удалось исправить в этой работе.

Заметим также, что для $I^{4}$ при использовании двоичного кодирования справедливо предложение 1 в новой следующей формулировке.

Предложение 2. Для каждого набора

$$
\alpha=\left(a_{1}, a_{2}, a_{3}, a_{4}, a_{5}, \ldots, a_{24}\right), \quad a_{i}=\{1,0\},
$$

соответствует одному из типов триангулящии $I^{4}$, существвет набор $\bar{\alpha}$, однозначно соответствующий тому же типу триангуляиии $I^{4}$.

Рассмотрим теперь задачу, аналогичную задаче разбиения $I^{3}$ на симплексы, - задачу разбиения $I^{4}$ на 4-симплексы. В данном случае будем проводить не произвольное 
разбиение на 4-симплексы, а поставим вопрос о том, существуют ли среди правильно триангулированных конфигураций $I^{4}$ правильные разбиения на 4 симплексы.

Сформулируем необходимое условие для выполнения такого разбиения.

Предложение 3. Для того, чтобы существовало правильное разбиение правильно триангулированных конфигураџий ${ }^{4}$ на 4-симплексы, необходимо, чтобы число 3-симплексов, входящих в развертку $I^{4}$, делилось наџело на 4.

Доказательство. Действительно, в 4-симплексе существует 4 трехмерные грани, представляющие собой 3-симплекс, поэтому необходимо, чтобы хотя бы число 3-симплексов в развертке $I^{4}$ было кратно 4.

Очевидно, что не все конфигурации $I^{4}$, характеризуемые векторами типов, представленные на графике распределения, удовлетворяют данному условию.

Рассмотрим все возможные конфигурации $I^{4}$. Для этого оценим сначала число 3-симплексов, входящих в каждый тип $I^{3}$.

(1) Тип $1-6$ симплексов.

(2) Тип $2-6$ симплексов.

(3) Тип $3-6$ симплексов.

(4) Тип $4-6$ симплексов.

(5) Тип 5-4 или 6 симплексов.

Таким образом, условию необходимости удовлетворяют все конфигурации $I^{4}$ в векторах, которых первые 4 типа присутствуют четное число раз и 5-й тип присутствует любое или соответственно четное число раз.

Попытаемся теперь на качественном уровне оценить возможность разбиения на 4-симплексы. В первую очередь это влечет, по аналогии с $I^{3}$ и с $I^{4}$, выделение ограниченного набора конфигураций, которые будут характеризовать тип триангуляции $I^{5}$. Однако, в действительности, разбиения $I^{4}$ на правильно расположенные 4-симплексы для предложенных разбиений на симплексы не существует. Но возможно построение неполных разбиений на 4-симплексы триангуляции $I^{4}$, например для конфигурации $(8,0,0,0,0)$.

Данный результат получен при помощи комбинаторных алгоритмов перебора вариантов разбиений на ЭВМ. При этом использовано представление триангулированного $I^{4}$ в виде бинарной матрицы связности, где 4-симплексу может быть сопоставлен некий набор из 1 и 0 из элементов матрицы связности.

Стоит отметить, что при использовании предложенного метода классификации, основанного на векторах числа типов, распределение триангуляций $I^{5}$ также можно описать статистической моделью Бозе-Эйнштейна. Это важное предположение позволяет говорить, что предложенный метод классификации является общим для правильной триангуляции $I^{n}$.

Приведенные выше результаты легко переносимы на широкий класс симплициальных комплексов, приводимых к кубическим комплексам.

Понятие приводимых симплициальных комплексов означает, что симплициальный комплекс может быть однозначно преобразован в кубический путем малых локальных деформаций без нарушения связности и выпуклости.

Малость локальных деформаций подразумевает, что любая вершина симплициального комплекса может перемещаться внутри сферы такого радиуса, который исключает 
возможность совпадения с любой другой вершиной и изменения положения вершины относительно гиперплоскости основания данного симплекса. Последнее требование обусловлено тем, что в процессе деформации не допускается взаимного пересечения граней симплекса кроме как по ребрам симплекса. Необходимым условием, для осуществления операции приведения является совпадение числа вершин симплициального комплекса с числом вершин в целевом кубическом комплексе, а также совпадение числа симплексов.

Таким образом, любой симплициальный комплекс, приводимый к кубическому, описывается при помощи соответствующего распределения.

\section{5. Заключение}

В заключении нужно сказать, что проводимая во всей работе параллель между двумерным, трехмерным, четырехмерным и пятимерным случаями имела целью показать схожесть рассматриваемых понятий и задач. В результате проведенных исследований получено распределение частот появления различных типов правильных триангуляций $I^{3}$ и $I^{4}$ при стохастической триангуляции пространства. Такой результат, благодаря выбранной структуре решетки дает возможность оценить поведение триангуляции во всем пространстве $\mathbf{Z}^{n}, n=3,4$. Установлено, что распределение по векторам числа типов для $I^{4}$ описывается статистической моделью Бозе-Эйнштейна. Также установлена невозможность построения на данных разбиениях $I^{4}$ разбиения на 4-симплексы, что затрудняет дальнейшее построение симплициальных разбиений высшей размерности для $I^{n}$.

\section{Список литературы}

1. Рябов Г. Г., Алгоритмические основы топологического процессора. В сб.: Tруды Bторой Bceроссийской научной конференции «Методы и средства обработки информации». ВМиК МГУ, Москва, 2005, с. 53-58.

2. Понтрягин Л. С., Основы комбинаторной топологии. Наука, Москва, 1986.

3. Мартинсон Л. К., Смирнов Е. В., Квантовая физика. Изд-во МГТУ им. Н. Э. Баумана, Москва, 2004.

4. Рябов Г. Г., Марковские процессы в динамике примитивных триангуляций в пространствах $\mathbf{R}^{2}$ и $\mathbf{R}^{3}$. Вычислительные методы и программирование (2009) 10, №1, 1-8.

Статья поступила 18.01.2011. 